\title{
Neonatal growth restriction-related leptin deficiency enhances leptin-triggered sympathetic activation and central angiotensin II receptor-dependent stress-evoked hypertension
}

Veronica Peotta' ${ }^{1}$ Kamal Rahmouni ${ }^{2,3}$, Jeffrey L. Segarr ${ }^{1}$, Donald A. Morgan², Kate M. Pitz' ${ }^{1}$ Olivia M. Rice ${ }^{1}$ and Robert D. Roghair ${ }^{1}$

BACKGROUND: Neonatal growth restriction ( $\mathrm{nGR}$ ) leads to leptin deficiency and increases the risk of hypertension. Previous studies have shown nGR-related hypertension is normalized by neonatal leptin (nLep) and exacerbated by psychological stress. With recent studies linking leptin and angiotensin signaling, we hypothesized that nGR-induced nLep deficiency increases adult leptin sensitivity; leading to leptin- or stressinduced hypertension, through a pathway involving central angiotensin II type 1 receptors.

METHODS: We randomized mice with incipient $n G R$, by virtue of their presence in large litters, to vehicle or physiologic nLep supplementation $(80 \mathrm{ng} / \mathrm{g} / \mathrm{d})$. Adult caloric intake and arterial pressure were monitored at baseline, during intracerebroventricular losartan infusion and during systemic leptin administration.

RESULTS: nGR increased leptin-triggered renal sympathetic activation and hypertension with increased leptin receptor expression in the arcuate nucleus of the hypothalamus; all of those nGR-associated phenotypes were normalized by nLep. nGR mice also had stress-related hyperphagia and hypertension, but only the stress hypertension was blocked by central losartan infusion.

CONCLUSION: nGR leads to stress hypertension through a pathway that involves central angiotensin II receptors, and nGR-associated leptin deficiency increases leptin-triggered hypertension in adulthood. These data suggest potential roles for preservation of neonatal growth and nLep supplementation in the prevention of nGR-related hypertension.

W orldwide, nearly $16 \%$ of all infants are born with low birth weight (1). Premature delivery and fetal growth restriction, the two predominant causes of low birth weight, both pose numerous and at times insurmountable challenges to the developing infant, including profound nutritional deficiencies. By the time premature infants reach $36 \mathrm{wk}$ postmenstrual age, nearly $90 \%$ have developed neonatal growth restriction (nGR) and up to $70 \%$ have elevated arterial pressure $(2,3)$. In one of the largest studies to date, Johansson and colleagues assessed blood pressure in 329,495 military conscripts and revealed a strong inverse association between birth weight and adult blood pressure, but the effect of intrauterine growth restriction was overshadowed by the consequences of preterm birth (4). Specifically, the odds of hypertension, adjusted for both birth weight and current body mass index, increased by 25,48 , and $93 \%$ in subjects born $4-7,8-11$, and $12-16$ wk prematurely, respectively (4).

As an adipose-derived neurotrophic hormone that is intimately involved in cardiometabolic regulation (5), leptin has emerged as a putative agent in both the inception and the propagation of programmed cardiovascular and metabolic dysfunction (6). Leptin readily crosses the placenta and typically exerts neurotrophic effects in the third trimester of fetal development. Preterm delivery abruptly terminates transplacental leptin delivery and preterm infants face the need to grow and develop in the presence of dramatic leptin deficiency $(7,8)$. Of those who survive this neonatal insult, the risk of adult cardiometabolic deterioration increases dramatically in the presence of adult obesity (9). Leptin levels strongly correlate with adult adiposity and investigations have revealed an important role for leptin in murine and human hypertension $(5,10)$, with relevant pathways including hypothalamic signaling, coactivation of the renin-angiotensin system and renal sympathetic nerve activation $(11,12)$.

In mice, the first $2 \mathrm{wk}$ of life model the third trimester of human neurodevelopment (13). Our previous investigations assessing the impact of naturally-occurring $\mathrm{nGR}$ revealed that nGR mice share many of the phenotypes described in former preterm infants, including leptin deficiency, postnatal growth restriction, neuropsychiatric impairment, and stress-evoked hypertension (14-16). Follow-up studies demonstrated normalization of brain morphology, neurodevelopment, and stress-associated (tail cuff) hypertension following neonatal leptin (nLep) supplementation $(14,16)$. We speculated that 


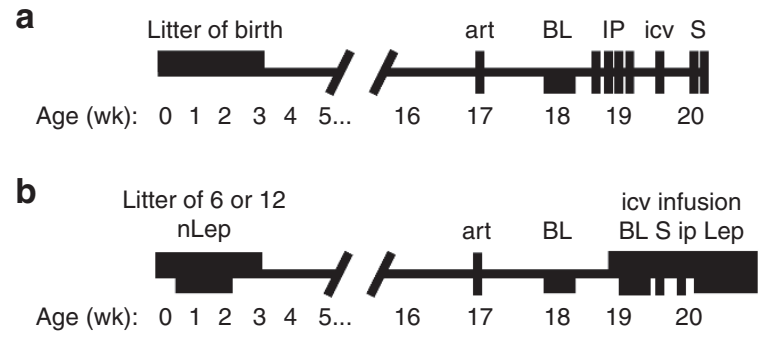

Figure 1. Protocol timeline. The first cohort of mice (a) were raised in their litter of birth. Utilizing the 10th percentile cutoff, neonatal growth restriction ( $\mathrm{nGR}$ ) pups were identified by a weight $<7.1 \mathrm{~g}$ at $3 \mathrm{wk}$. Once they reached $17 \mathrm{wk}$, a radiotelemetry-enabled carotid artery catheter was placed (art). After $1 \mathrm{wk}$ of recovery, hemodynamics were measured throughout a $60 \mathrm{~h}$ baseline window (BL). This was followed by a series of four randomized once daily intraperitoneal injections including vehicle alone, scopolamine, chlorisondamine, and prazosin (IP). Mice then underwent icv cannulation followed in $48 \mathrm{~h}$ by placement in a stress-evoking metabolic cage (S), first without losartan pretreatment and then $24 \mathrm{~h}$ later, with losartan pretreatment. The second cohort of mice (b) were fostered in litters of 6 or 12 pups with those in litters of 12 randomized to either vehicle or nLep on days 4 to 14. The same weight-based criteria were utilized to distinguish control and nGR mice and carotid catheters were again placed and $B L$ readings were obtained. This time, the $B L$ recordings were immediately followed by icv cannulation for $14 \mathrm{~d}$ continuous infusion of artificial cerebrospinal fluid or losartan. After $48 \mathrm{~h}$ recovery, new BL recordings were obtained, followed the next day by $S$ recordings that were in turn followed by recordings during ip challenge with vehicle or leptin. Finally, on days 10 to 13 of icv infusion, the mice received twice daily intraperitoneal leptin (Lep). Additional mice fostered by the second breeding approach did not undergo radiotelemeter implantation, but rather were used for sympathetic recordings or gene expression analysis.

nGR programs a range of adverse developmental outcomes by persistently upregulating hypothalamic leptin responsiveness.

With recent evidence linking leptin with the hemodynamic response to psychological stress and the central renin-angiotensin system $(11,12)$, we sought to test the hypothesis that nGR leads to stress- or leptin-evoked hypertension in a pathway that involves the angiotensin II type 1 receptor $\left(A_{1} R_{1}\right)$. In order to isolate the cardiovascular phenotypes programmed in $\mathrm{nGR}$ mice, we incorporated an extensive array of advanced physiologic methods, including carotid radiotelemetry, renal sympathetic nerve recordings and intracerebroventricular infusions (Figure 1). Once the nGR-induced phenotypes were identified, we tested the rigorous hypothesis that nGR mice not only did not significantly differ from control mice (either because of similarity or a lack of statistical power), but were statistically equivalent to control mice with cardiovascular phenotypes constrained below the levels seen in $\mathrm{nGR}$ mice that had not received nLep supplementation.

\section{RESULTS}

By definition, nGR mice had reduced weanling weights, and this was independent of nLep therapy (Table 1). Overall 10.3\% (20 of 195) male mice fostered in standardized litters of 6 pups developed nGR, as defined by a weanling weights below our historic 10th percentile cut-off of $7.1 \mathrm{~g}$. The occurrence of nGR increased to $40.2 \%$ (131 of 326) for mice fostered in litters of 12 pups. As adults, nGR mice remained smaller than controls, and
Table 1. Baseline body weight and ambulatory hemodynamic data

\begin{tabular}{lccc}
\hline & $\begin{array}{c}\text { Control } \\
(n=30)\end{array}$ & $\begin{array}{c}\text { nGR } \\
(n=25)\end{array}$ & $\begin{array}{c}\text { nGR-nLep } \\
(n=22)\end{array}$ \\
\hline Weight, $20 \mathrm{~d}(\mathrm{~g})$ & $8.7 \pm 0.2$ & $6.3 \pm 0.1^{*}$ & $6.3 \pm 0.2^{*}$ \\
Weight, adult (g) & $32.0 \pm 1.0$ & $28.0 \pm 0.8^{*}$ & $30.1 \pm 1.0$ \\
SBP (mmHg) & $128 \pm 1$ & $128 \pm 2$ & $126 \pm 4$ \\
DBP (mmHg) & $96 \pm 1$ & $96 \pm 2$ & $93 \pm 3$ \\
HR (bpm) & $556 \pm 6$ & $568 \pm 7$ & $561 \pm 9$ \\
Activity (au) & $4.4 \pm 0.3$ & $4.7 \pm 0.4$ & $4.7 \pm 0.3$
\end{tabular}

${ }^{*} P<0.01$ vs. control; au = arbitrary units; 18 control and $11 \mathrm{nGR}$ mice were fostered by their birth dam without neonatal injections; 12 control, 14 nGR and all nGR-nLep mice were cross-fostered by an adoptive dam with neonatal injections.

DBP, diastolic blood pressure; HR, heart rate; $\mathrm{nGR}$, neonatal growth restriction; $\mathrm{SBP}$, systolic blood pressure.

the null hypothesis that $\mathrm{nGR}$ and control mice did not differ was rejected $(P<0.01)$. However, the $90 \%$ confidence interval for the difference in means between nGR-nLep and control mice $(-4.3$ to $0.5 \mathrm{~g})$ extended beyond the mean difference between nGR and control mice $(-4.0 \mathrm{~g})$, leading to acceptance of the null hypothesis that control and nGR-nLep supplemented mice may differ as much as control and nGR mice that did not receive nLep (Table 1). Beyond body weight, baseline ambulatory blood pressure, heart rate, and locomotor activity were not significantly altered by nGR (Table 1). Comparing the two cohorts of mice that subsequently underwent different experimental protocols (Figure 1), adult weight and hemodynamic parameters were independent of whether the mice were fostered by their birth dam without neonatal injections (cohort 1) or cross-fostered by an adoptive dam with neonatal injections (cohort 2) (data not shown).

Given investigations revealing growth restriction-associated increases in sympathetic tone (17), mice were subsequently challenged with a series of autonomic antagonists. Consistent with a predominance of resting cardiac sympathetic activation in mice, scopolamine did not significantly alter either blood pressure or heart rate, while the ganglionic blocker chlorisondamine caused a dramatic reduction in both parameters, independent of nGR status (Figure 2). Finally, the $\alpha_{1}$-adrenergic receptor selective antagonist prazosin elicited a comparable hypotensive response in both $\mathrm{nGR}$ and control mice (Figure 2).

To begin assessment of central autonomic regulation, intracerebroventricular (icv) catheters were placed and hemodynamics were measured during psychologic challenge. Compared to control mice, nGR mice had an exaggerated hypertensive response to cage switch stress, and icv losartan significantly reduced the stress-evoked hypertension displayed by nGR mice (Figure 2). Independent of nGR status, cage-switch stress led to a near doubling corticosterone levels (control baseline $137 \pm 23 \mathrm{ng} / \mathrm{ml}$ vs. stressed $250 \pm 19 \mathrm{ng}$ / $\mathrm{ml}, P<0.01, n=7$; nGR baseline $132 \pm 13 \mathrm{ng} / \mathrm{ml}$ vs. stressed $224 \pm 24 \mathrm{ng} / \mathrm{ml}, P<0.01, n=6$ ), confirming the presence of a stress response, but suggesting the exaggerated response seen in nGR mice was not driven by a programmed change in corticosterone production. 


\section{Articles $\mid$ Peotta et al.}
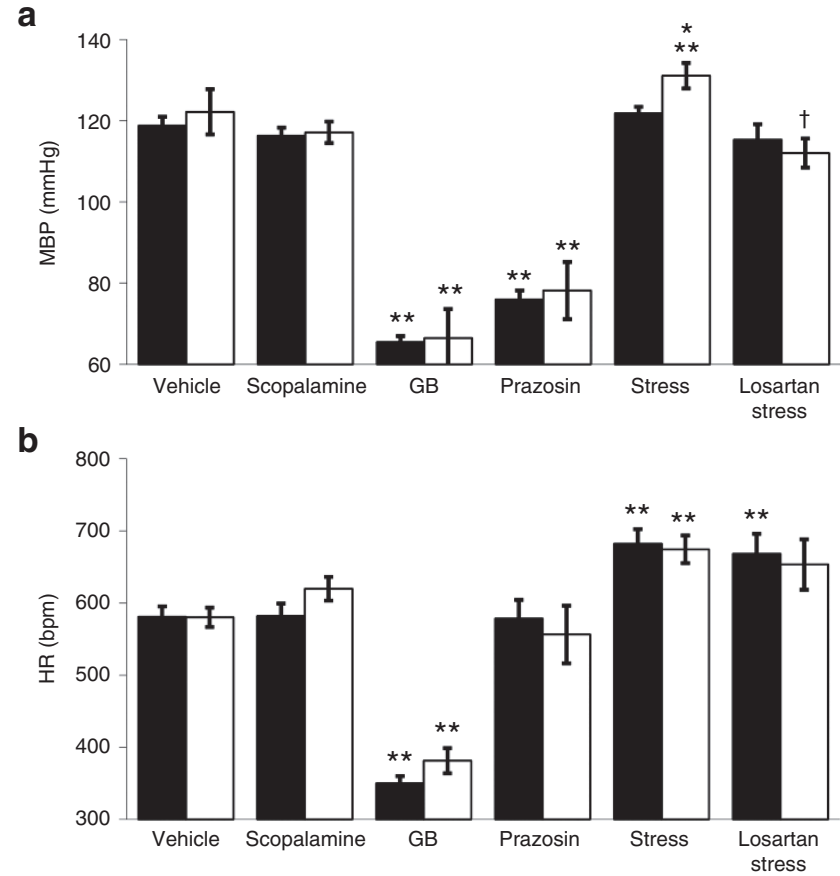

Figure 2. Hemodynamic responses to autonomic blockers or psychological stress. Mean blood pressure (MBP, a) and heart rate $(\mathrm{HR}, \mathbf{b})$ were measured 20-50 min following randomized intraperitoneal injections of normal saline (vehicle, $10 \mathrm{ml} / \mathrm{kg}$ ), scopolamine $(2 \mathrm{mg} / \mathrm{kg})$, the ganglionic blocker chlorisondamine (GB, $2.5 \mathrm{mg} / \mathrm{kg})$, or prazosin $(1 \mathrm{mg} / \mathrm{kg})$. Following those injections, an icv catheter was placed and measurements were obtained during placement in a stress-evoking metabolic cage, without and with losartan pretreatment $(20 \mu \mathrm{g} i \mathrm{cv})$. For both control (solid bars) and neonatal growth restriction (nGR) mice (open bars), GB lowered MBP and HR while prazosin lowered MBP and placement in a metabolic cage increased HR. Compared to control mice, nGR mice had stressevoked hypertension that was blocked by losartan. ${ }^{*} P<0.05$ vs. control, ${ }^{*} P<0.05$ vs. normal saline, $+P<0.05$ vs. no losartan; $n=11$ control and 7 GR ( 1 control mouse did not receive the intraperitoneal injections; 2 control mice did not have post-icv losartan data including 1 mouse that did not undergo icv cannulation and 1 mouse that died during icv injection; 3 GR mice did not have post-icv losartan data including 1 mouse that did not undergo cannulation, 1 mouse that died during icv injection and 1 mouse that lost pulsatile arterial pressure readings with a MBP consistently $<60 \mathrm{mmHg}$ following icv injection).

The second cohort of mice was then utilized to determine whether physiologic nLep and/or continuous $\mathrm{AT}_{1} \mathrm{R}$ antagonist administration protect nGR mice from stress-evoked hypertension. Once again nGR mice receiving only an infusion of artificial cerebrospinal fluid (aCSF) weighed less than their control counterparts (mean difference of $-4.6 \mathrm{~g}$, Figure 3a), and that was again not definitively prevented by nLep (90 percent confidence interval for the mean difference between nGR-nLep and control: -9.4 to $0.2 \mathrm{~g}$ ). Unexpectedly, icv cannulation with infusion of either aCSF or losartan dramatically increased the food intake of nGR-vehicle mice (Figure $3 \mathbf{b}$ ), raising the possibility of increased stress reactivity. Independent of aCSF vs. losartan administration, both control and nGR mice lost weight during twice daily leptin administration (Figure 3a), and that was associated with a leptin-induced anorexia in all but nGR mice receiving icv losartan (Figure 3b).
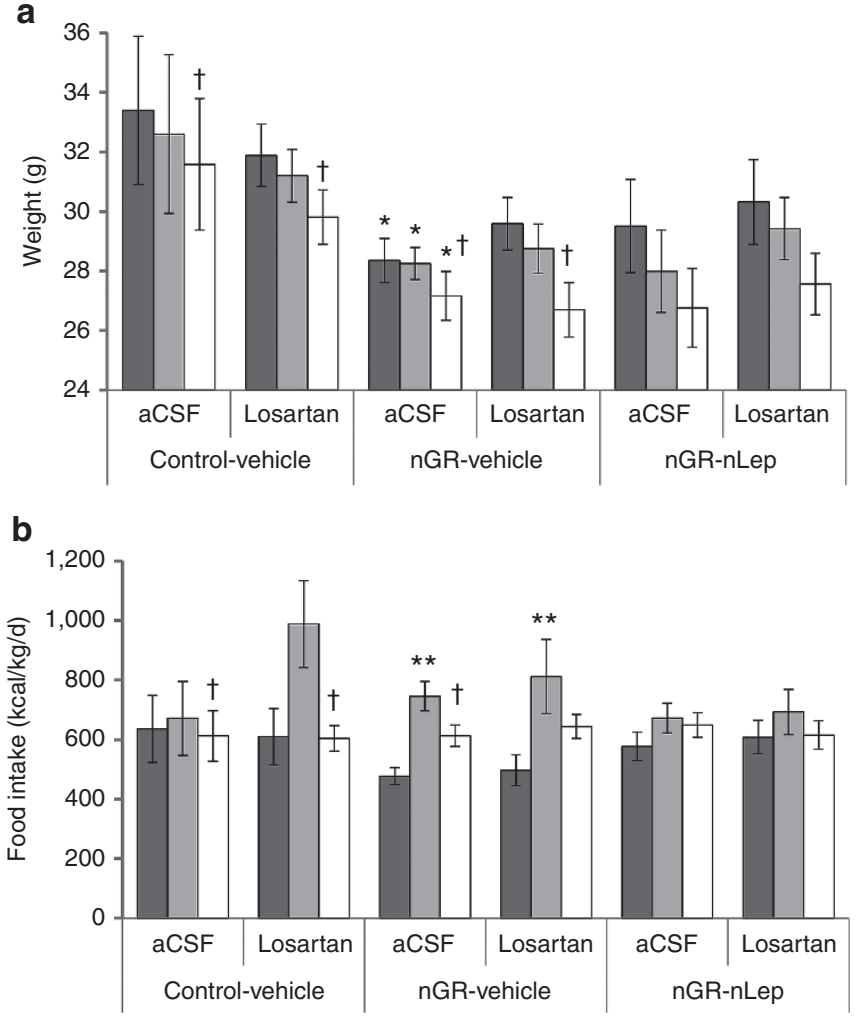

Figure 3. Longitudinal body weight and food intake. Weight (a) and food intake (b) were sequentially collected prior to icv cannulation (baseline, dark gray bars), during icv infusion of artificial cerebrospinal fluid (aCSF) or losartan (light gray bars) and during twice daily intraperitoneal leptin administration (open bars). ${ }^{*} P<0.05$ vs. control, ${ }^{* *} P<0.05$ vs. baseline, $+P<0.05$ vs. no leptin. $n=6$ other than 10 for nGR-nLep with aCSF and 11 for nGR-nLep with losartan. nGR, neonatal growth restriction.

Regarding hemodynamic outcomes, nGR and low dose central losartan infusion had no significant effect on resting blood pressure, heart rate, or locomotor activity beyond a mild losartan-induced decrease in systolic blood pressure that was exclusively seen in control mice (Table 2). A significant interaction between nGR and losartan administration influenced the hypertensive response to stress (Figure 4). Compared to the corresponding controls (Figure 4), nGR mice receiving aCSF had an exaggerated hypertensive response to placement in the metabolic cage (mean difference of 3.7\%), and that stress response was significantly suppressed by icv losartan, but not normalized to equivalence by nLep ( 90 percent confidence interval for the mean difference between nGR-nLep and control: -10 to $6.7 \%)$.

To further test our hypothesis that nGR-associated nLep deficiency increases adult leptin responsiveness through a pathway involving the $\mathrm{AT}_{1} \mathrm{R}$, we went on to assess the independent and interactive effects of nGR and central losartan administration on the hemodynamic responses evoked by leptin. Unlike control mice, nGR-vehicle mice had an exaggerated hypertensive response to the initial dose of exogenous leptin (mean difference in systolic, mean and diastolic pressures after leptin vs. vehicle administration of 10.5, 9.2, and $8.2 \mathrm{mmHg}$, respectively), and those 
responses were absent in $\mathrm{nGR}$ mice that were receiving $\mathrm{icv}$ losartan (Table 3). The hemodynamic responses of nGRnLep mice to exogenous leptin were diametrically opposed to the responses seen in nGR-vehicle mice and the systolic blood pressure response was statistically equivalent to the response of control mice (confidence intervals for the difference between control and nGR-nLep mice for systolic, mean, and diastolic pressures were -9.0 to $1.0,-14.6$ to 0.9 , and -17.4 to 2.3 ) (Table 3 ).

To determine if the pressor response to leptin is sustained during ongoing leptin administration, mice were next given twice daily leptin for 4 consecutive days. Compared to the baseline results obtained during icv infusion of aCSF or losartan in the absence of exogenous leptin, sustained leptin administration only elicited a mild increase in mean and diastolic blood pressure among control mice receiving icv losartan infusion (Table 4). This contrasts with the persistent leptin-induced

Table 2. Radiotelemetry during central infusion of the aCSF vehicle or the angiotensin II receptor antagonist losartan

\begin{tabular}{|c|c|c|c|c|c|c|}
\hline & \multicolumn{2}{|c|}{ Control-vehicle } & \multicolumn{2}{|c|}{ nGR-vehicle } & \multicolumn{2}{|c|}{ nGR-nLep } \\
\hline & $\begin{array}{l}\mathrm{aCSF} \\
(n=6)\end{array}$ & $\begin{array}{l}\text { Losartan } \\
(n=5)\end{array}$ & $\begin{array}{l}\text { aCSF } \\
(n=5)\end{array}$ & $\begin{array}{c}\text { Losartan } \\
(n=8)\end{array}$ & $\begin{array}{c}\mathrm{aCSF} \\
(n=10)\end{array}$ & $\begin{array}{c}\text { Losartan } \\
(n=9)\end{array}$ \\
\hline $\mathrm{SBP}(\mathrm{mmHg})$ & $132 \pm 4$ & $124 \pm 3$ & $126 \pm 2$ & $131 \pm 4$ & $128 \pm 4$ & $126 \pm 4$ \\
\hline (\% baseline) & $101 \pm 1$ & $95 \pm 3^{* *}$ & $100 \pm 1$ & $100 \pm 1$ * & $100 \pm 2$ & $97 \pm 3$ \\
\hline $\mathrm{MBP}(\mathrm{mmHg})$ & $115 \pm 3$ & $110 \pm 3$ & $110 \pm 2$ & $114 \pm 3$ & $110 \pm 4$ & $109 \pm 3$ \\
\hline (\% baseline) & $101 \pm 1$ & $96 \pm 3$ & $101 \pm 1$ & $100 \pm 1$ & $99 \pm 2$ & $96 \pm 3$ \\
\hline $\mathrm{DBP}(\mathrm{mmHg})$ & $97 \pm 4$ & $97 \pm 4$ & $94 \pm 3$ & $97 \pm 3$ & $92 \pm 3$ & $91 \pm 2$ \\
\hline (\% baseline) & $100 \pm 1$ & $99 \pm 3$ & $102 \pm 2$ & $100 \pm 1$ & $98 \pm 2$ & $95 \pm 3$ \\
\hline $\mathrm{HR}$ (bpm) & $569 \pm 17$ & $555 \pm 10$ & $576 \pm 5$ & $591 \pm 9$ & $574 \pm 16$ & $594 \pm 10$ \\
\hline (\% baseline) & $103 \pm 2$ & $101 \pm 2$ & $101 \pm 1$ & $102 \pm 1$ & $103 \pm 2$ & $103 \pm 1$ \\
\hline Activity (au) & $7.0 \pm 0.8$ & $4.9 \pm 0.4$ & $5.9 \pm 0.9$ & $6.2 \pm 0.9$ & $5.2 \pm 0.4$ & $6.0 \pm 0.6$ \\
\hline (\% baseline) & $149 \pm 24$ & $102 \pm 18$ & $116 \pm 27$ & $117 \pm 7$ & $120 \pm 6$ & $127 \pm 17$ \\
\hline
\end{tabular}

a

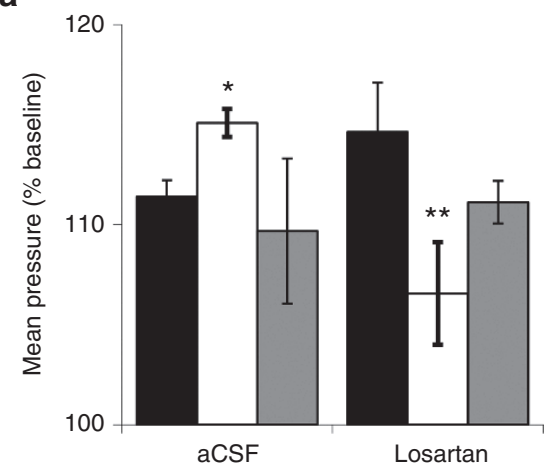

anorexia seen in nGR-vehicle mice during that same treatment period (Figure $3 \mathbf{b}$ ).

In order to further evaluate the role of acute leptin exposure on nGR-associated sympathetic activation, renal sympathetic nerve recordings were obtained during intravenous leptin administration. Throughout the second hour of recording, leptin-evoked changes in renal sympathetic nerve activity (RSNA) were significantly increased in nGR mice (Figure 5a, mean differences vs. control of 23 to $30 \%$ ). The leptin-evoked RSNA of nGR-nLep mice were again diametrically opposed to the responses seen in nGRvehicle mice and statistically equivalent to the responses of control mice at 60 and $75 \mathrm{~min}$ (Figure 5a, confidence intervals for the difference between control and nGR-nLep mice ranged from -22 to 15 at $60 \mathrm{~min}$ to -54 to 13 at $120 \mathrm{~min}$ ). During the RSNA recordings, blood pressure consistently decreased (Figure 5b) and heart rate consistently increased (Figure 5c). The absence of leptin-triggered hypertension during anesthetized RSNA recording is consistent with results previously reported with this method in mice (18). Given the possibility that leptin-dependent NOS activation may partially buffer the hemodynamic effects of leptin, the nitric oxide synthase antagonist L-NAME was administered at the end of the protocol. L-NAME rapidly and dramatically increased arterial pressure with an exaggerated hypertensive response seen in nGR mice (Figure 5d, mean difference vs. control at $3 \mathrm{~min}$ : 37\%) that was not normalized by nLep (confidence interval for the difference between control and nGR-nLep mice: 5.1 to $45 \%$ ).

Precision biopsies of the hypothalamic regions that are critical in mediating leptin's hemodynamic effects were obtained and both LepR and $\mathrm{AT}_{1} \mathrm{R}$ mRNA expression were analyzed by qPCR. Unlike expression in the ventromedial hypothalamus, LepRb expression in the arcuate nucleus of the hypothalamus (ARC) was significantly increased in nGR mice (Figure 6, mean difference vs. control: 86\%), and this was normalized by nLep (confidence interval for the difference between control and nGR-nLep mice: -53 to $62 \%$ ). There were no corresponding alterations in the expression of either the signaling-deficient short form of the leptin receptor (LepRa) or the $\mathrm{AT}_{1} \mathrm{R}$ (Figure 6). b

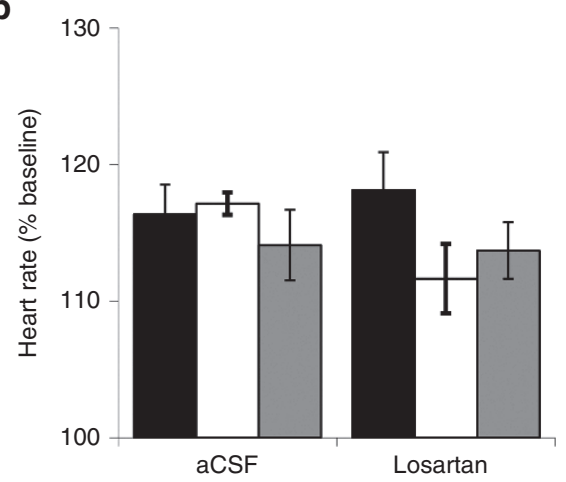

Figure 4. Hemodynamic responses to cage switch stress. During continuous infusion of either artificial cerebrospinal fluid (aCSF) or losartan (2.5 $\mu \mathrm{g} / \mathrm{h})$, mice were placed in a metabolic cage and arterial pressure (a) and heart rate (b) were recorded for $60 \mathrm{~min}$, with the results normalized to the values obtained during the post-icv cannulation baseline. Cage switch evoked a significantly greater pressor response in neonatal growth restriction (nGR)-vehicle mice (white bars, $n=5$ aCSF, $n=8$ losartan) than control mice (black bars, $n=6 \mathrm{aCSF}, n=5$ losartan), a response that was not significantly altered by $n L e p$ supplementation (nGR-nLep mice, gray bars, $n=10 \mathrm{aCSF}, n=9$ losartan), but was blocked by central losartan administration. ${ }^{*} P<0.01$ vs. control, ${ }^{* *} P<0.05$ vs. aCSF. 
Table 3. Acute leptin-evoked hemodynamic responses, in relation to the results obtained during administration of the normal saline vehicle alone, all in the presence of ongoing central infusion of aCSF or losartan

\begin{tabular}{|c|c|c|c|c|c|c|}
\hline & \multicolumn{2}{|c|}{ Control } & \multicolumn{2}{|c|}{ nGR } & \multicolumn{2}{|c|}{ nGR-nLep } \\
\hline & $\begin{array}{l}\mathrm{aCSF} \\
(n=6)\end{array}$ & $\begin{array}{c}\text { Losartan } \\
(n=5)\end{array}$ & $\begin{array}{l}\mathrm{aCSF} \\
(n=5)\end{array}$ & $\begin{array}{c}\text { Losartan } \\
(n=6)\end{array}$ & $\begin{array}{c}\mathrm{aCSF} \\
(n=10)\end{array}$ & $\begin{array}{c}\text { Losartan } \\
(n=9)\end{array}$ \\
\hline $\mathrm{SBP}(\mathrm{mmHg})$ & $128 \pm 3$ & $119 \pm 7$ & $130 \pm 1$ & $122 \pm 4$ & $122 \pm 4$ & $119 \pm 3$ \\
\hline (\% vehicle) & $104 \pm 4$ & $101 \pm 6$ & $109 \pm 2^{*}$ & $99 \pm 3$ & $97 \pm 2^{+}$ & $100 \pm 3$ \\
\hline $\mathrm{MBP}(\mathrm{mmHg})$ & $110 \pm 2$ & $106 \pm 6$ & $114 \pm 2$ & $106 \pm 3$ & $104 \pm 4$ & $104 \pm 2$ \\
\hline (\% vehicle) & $104 \pm 4$ & $101 \pm 6$ & $109 \pm 2^{*}$ & $99 \pm 3$ & $94 \pm 3$ & $100 \pm 3$ \\
\hline $\mathrm{DBP}(\mathrm{mmHg})$ & $91 \pm 3$ & $94 \pm 7$ & $97 \pm 2$ & $89 \pm 3$ & $86 \pm 4$ & $88 \pm 3$ \\
\hline (\% vehicle) & $104 \pm 4$ & $100 \pm 6$ & $110 \pm 2^{*}$ & $98 \pm 3$ & $93 \pm 4$ & $99 \pm 2$ \\
\hline $\mathrm{HR}$ (bpm) & $540 \pm 11$ & $558 \pm 15$ & $571 \pm 16$ & $552 \pm 14$ & $544 \pm 25$ & $584 \pm 10$ \\
\hline (\% vehicle) & $104 \pm 5$ & $97 \pm 3$ & $108 \pm 1$ & $96 \pm 2^{* *}$ & $91 \pm 3$ & $102 \pm 2$ \\
\hline
\end{tabular}

${ }^{*} P<0.05$ vs. vehicle. ${ }^{* *} P<0.05$ vs. aCSF. $+P<0.05$ for equivalence to control. aCSF, artificial cerebrospinal fluid; DBP, diastolic blood pressure; $H R$, heart rate; $M B P$, mean blood pressure; nGR, neonatal growth restriction; SBP, systolic blood pressure.

Table 4. Hemodynamic status during prolonged twice daily leptin administration, in relation to the values obtained during central aCSF or losartan infusion prior to exogenous leptin administration

\begin{tabular}{lccccccc}
\hline & \multicolumn{2}{c}{ Control } & & \multicolumn{2}{c}{ nGR } & & \multicolumn{2}{c}{ nGR-nLep } \\
\cline { 2 - 3 } & $\begin{array}{c}\text { aCSF } \\
(n=6)\end{array}$ & $\begin{array}{c}\text { Losartan } \\
(n=5)\end{array}$ & $\begin{array}{c}\text { aCSF } \\
(n=5)\end{array}$ & $\begin{array}{c}\text { Losartan } \\
(n=6)\end{array}$ & $\begin{array}{c}\text { aCSF } \\
(n=10)\end{array}$ & $\begin{array}{c}\text { Losartan } \\
(n=9)\end{array}$ \\
\hline SBP (mmHg) & $129 \pm 4$ & $127 \pm 4$ & $127 \pm 4$ & $127 \pm 3$ & $122 \pm 5$ & $120 \pm 5$ \\
(\% baseline) & $97 \pm 1$ & $102 \pm 2$ & $101 \pm 2$ & $101 \pm 1$ & $96 \pm 3$ & $96 \pm 2$ \\
MBP (mmHg) & $112 \pm 3$ & $113 \pm 2$ & $110 \pm 3$ & $111 \pm 2$ & $105 \pm 5$ & $105 \pm 4$ \\
(\% baseline) & $97 \pm 1$ & $102 \pm 2^{* *}$ & $100 \pm 2$ & $100 \pm 1$ & & $95 \pm 3$ & $96 \pm 2$ \\
DBP (mmHg) & $95 \pm 3$ & $99 \pm 2$ & $93 \pm 2$ & $94 \pm 2$ & & $87 \pm 4$ & $88 \pm 3$ \\
(\% baseline) & $97 \pm 1$ & $103 \pm 2^{* *}$ & $98 \pm 1$ & $101 \pm 1$ & $94 \pm 3$ & $97 \pm 2$ \\
HR (bpm) & $558 \pm 6$ & $554 \pm 8$ & $569 \pm 11$ & $583 \pm 5^{*}$ & $566 \pm 17$ & $575 \pm 8$ \\
(\% baseline) & $98 \pm 2$ & $100 \pm 2$ & $99 \pm 2$ & $97 \pm 1$ & $99 \pm 2$ & $97 \pm 2$ \\
\hline
\end{tabular}

${ }^{*} P<0.05$ vs. control. ${ }^{* *} P<0.05$ vs. Baseline.

aCSF, artificial cerebrospinal fluid; DBP, diastolic blood pressure; HR, heart rate; MBP, mean blood pressure; nGR, neonatal growth restriction; SBP, systolic blood pressure.

\section{DISCUSSION}

The inverse relationship between growth through $2 \mathrm{y}$ and blood pressure in adulthood is a prototypic example of undernutrition during a critical window of development predisposing individuals to adult disease (19). Animal models have been essential in the identification of the pathways that lead to programmed hypertension and may be amenable to therapeutic intervention. Among the potential interacting pathways, an important role for angiotensin receptor activation has been suggested in studies from diverse programming models. Global maternal undernutrition directly enhances cardiovascular responses to angiotensin II in the rat (20). Likewise, maternal dexamethasone enhances the blood pressure response of adult sheep to icv angiotensin II and central losartan normalizes the programmed hypertension (21). Our present investigations are the first we are aware of that investigated the interaction
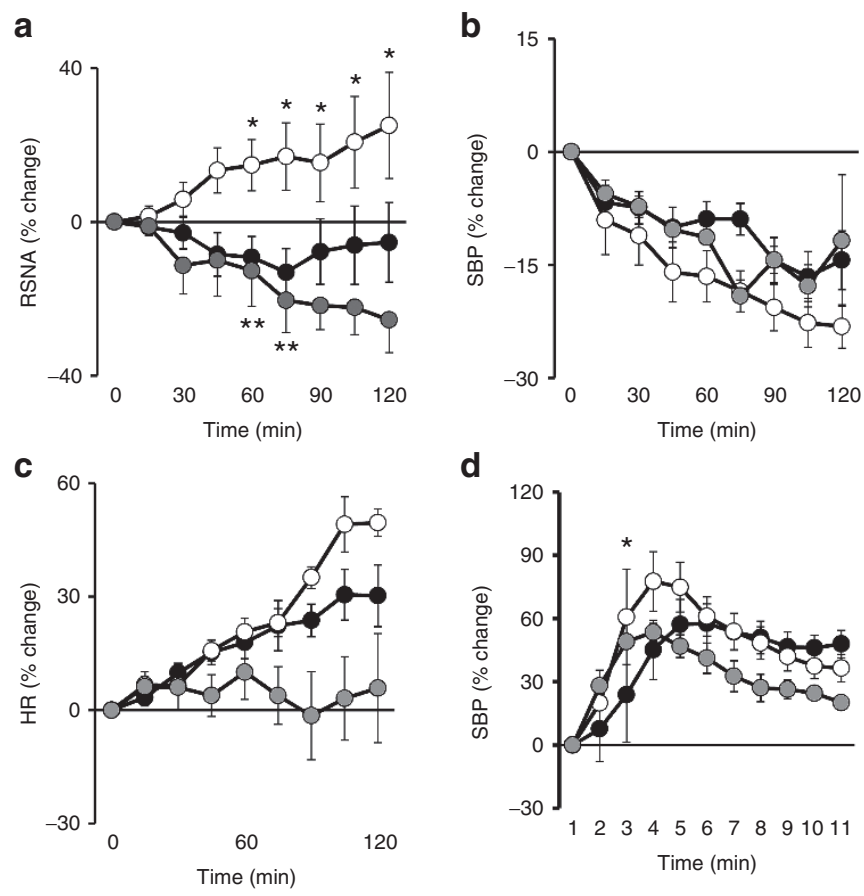

d

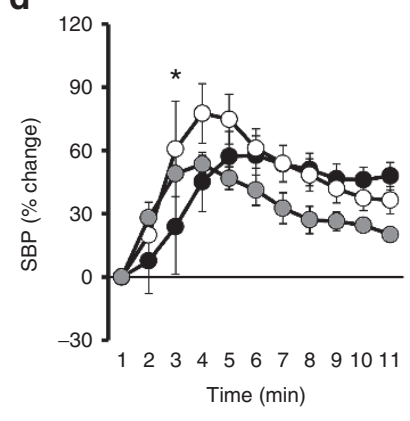

Figure 5. RSNA following intravenous leptin. RSNA (a), systolic blood pressure $(\mathrm{SBP}, \mathbf{b})$, and heart rate $(\mathrm{HR}, \mathbf{c})$ were simultaneously recorded following intravenous leptin administration. After the $2 \mathrm{~h}$ recordings, the nitric oxide synthase inhibitor L-NAME was administered and SBP was monitored an additional $10 \mathrm{~min}$ (d). Compared to control mice (black symbols, $n=12$ ), neonatal growth restriction ( $\mathrm{GGR}$ ) mice (white symbols, $n=11$ ) but not $n G R-n L e p$ mice (gray symbols, $n=4$ ) had exaggerated RSNA responses to leptin. ${ }^{*} P<0.05$ vs. control, ${ }^{*} P<0.05$ for equivalence to control.

between leptin and angiotensin receptors in the programming of central cardiovascular regulation.

We have significantly extended our earlier investigations showing that perinatal growth restriction leads to reduced adult weight- and stress-related hypertension (22). Our present studies have identified associations between increased ARC leptin receptor expression and heightened stress or leptin-evoked blood pressure responses in nGR mice. The presence of relatively normal ambulatory blood pressure with exaggerated responses to psychological stress is consistent with human epidemiological data, as well as our prior investigations utilizing a stress-evoking tail cuff blood pressure monitoring system (23). While previous studies have shown losartan potently inhibits psychological stressinduced hypertension (24), the additional finding that central losartan administration decreases the response of nGR-vehicle mice to systemically administered leptin is unique. The increase in nGR-vehicle mouse food intake observed following icv cannulation was dramatic and may be related to the stress associated with the procedure. If this is the case, stress-induced hyperphagia may contribute to the increased central adiposity seen in a subpopulation of growth-restricted individuals, (25) which otherwise remain smaller than their normal birth weight peers (26).

Beyond the ability of central AT ${ }_{1}$ blockade to prevent the development of stress-evoked hypertension, $\mathrm{AT}_{1} \mathrm{R}$ inhibition interfered with the hypertension evoked by leptin, but not the 


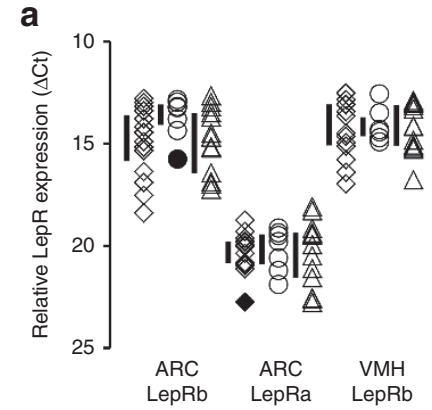

b
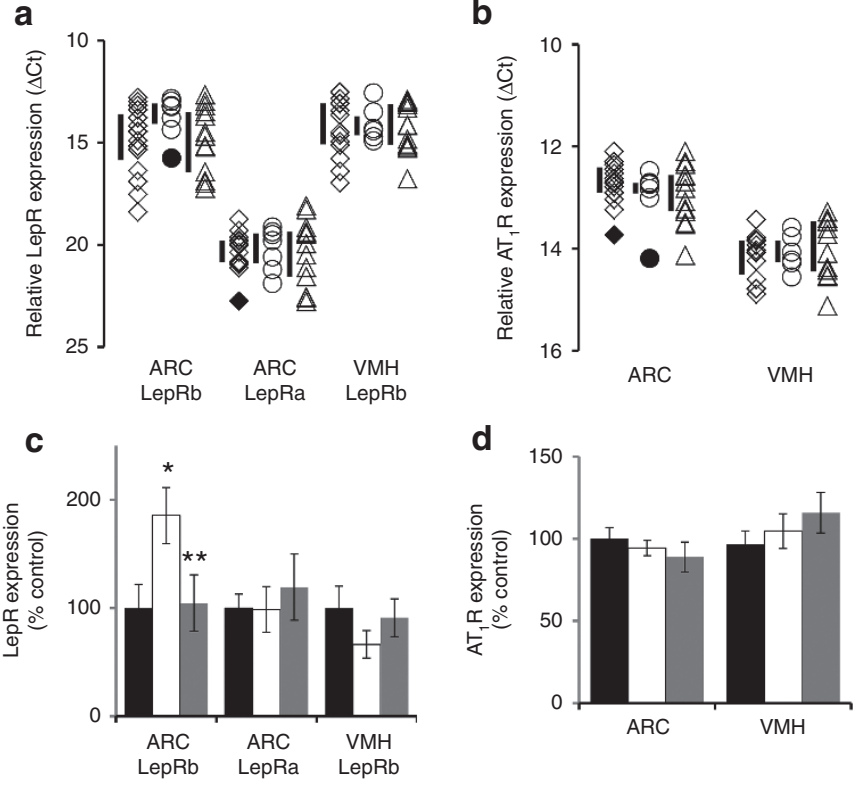

Figure 6. Impact of neonatal growth restriction ( $n G R)$ and $n L e p$ on leptin receptor and angiotensin II receptor expression with the hypothalamus. Expression within the ARC and ventromedial hypothalamus (VMH) of the leptin receptor (a, long-form: LepRb; short-form: LepRa) and AT $R$ (b) was determined for adult control (rhombi or black bars, $n=15$ for ARC, $n=14$ for VMH), nGR (circles or white bars, $n=7$ for ARC, $n=6$ for VMH) and nGRnLep mice (triangles or gray bars, $n=13$ for ARC, $n=12$ for VMH). Four values that were greater than 1.5 times the span of the interquartile range outside of the interquartile range were a priori excluded as statistical outliers (solid symbols). Relative mRNA expression was then compared by

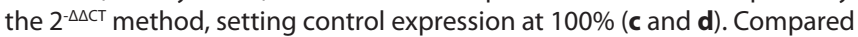
to control mice LepRb mRNA expression was increased in the ARC of nGR but not nGR-nLep mice. ${ }^{*} P<0.05$ vs. control, ${ }^{* *} P<0.05$ for equivalence to control.

anorexia response to leptin, suggesting alternative pathways are involved in leptin's cardiovascular and metabolic effects (27). Because leptin-induced RSNA is known to be dependent on downstream AT $\mathrm{R}_{1}$ signaling (12), we did not investigate the effect of losartan on leptin-evoked RSNA in the present study.

Given investigations in humans suggesting increased sympathetic tone and heightened stress reactivity in growth-restricted individuals $(17,28)$, we investigated the effects of nGR with or without nLep administration on adult leptin-evoked RSNA. nGR mice that did not receive nLep had an increased RSNA response within $2 \mathrm{~h}$ of leptin administration. Throughout that time, all mice became progressively hypotensive and tachycardic. Those hemodynamic changes are consistent with the findings previously reported in mice that received icv leptin (12). While the declining blood pressures were potentially a manifestation of prolonged anesthesia, we were intrigued by publications showing leptin upregulates vascular nitric oxide synthase to buffer the development of hypertension that would be expected from parallel sympathetic activation (29). Our data demonstrating a gradual decrease in blood pressure during RSNA recording followed by a rapid and robust increase in arterial pressure after L-NAME administration are consistent with leptin-triggered nitric oxide production in control and nGR mice. Further studies are needed to elucidate whether the relative hypertension seen in nGR mice following leptin plus L-NAME is a reflection of the increased RSNA or an increased reliance on the vasodilatory effects of nitric oxide. Our prior investigations in complementary nGR models support the presence of enhanced vascular nitric oxide-mediated vasodilatation in otherwise hypertensive mice (30). It is possible that exaggerated nitric oxide production attenuated the response of nGR mice to prolonged leptin administration; further investigation is needed to determine if that possible compensatory response is lost with aging, obesity, or other mediators of endothelial dysfunction. Additional investigations, including sympathetic blockade or baroreceptor denervation, are likewise needed to more definitively identify sympathetic activation as a requisite for nGR-related hypertension.

Taken together, our data support a critical interaction between leptin and central $\mathrm{AT}_{1} \mathrm{R}$ in programmed cardiovascular dysregulation. Regarding brain-site-specific regulation, studies in transgenic mice lacking leptin receptors within the ARC have solidified the prime importance of the ARC in leptin's sympathetic and pressor effects (31). Among leptinresponsive neuronal regions, the ARC is also exquisitely sensitive to environmental modulation of leptin sensitivity (32), perhaps explaining the selective alteration in leptin receptor mRNA expression we noted within the ARC in the current studies. While intravenous administration was utilized to determine the physiologically relevant effects of systemically delivered leptin, additional mechanistic studies could include further assessment of leptin receptor splice variants and icv leptin administration to determine if the increased response seen in nGR mice is related to enhanced access to central leptin receptors. In addition to leptin, insulin signaling within the ARC plays an important role in diabetesrelated sympathetic activation (33). With peripheral insulin resistance common in former growth-restricted individuals, the potential for selective central insulin sensitization merits further investigation.

nLep replacement is a more targeted therapy than global overnutrition and may serve to rescue cardiovascular health while avoiding the increased risk of obesity and obesity-related hyperleptinemia plus endothelial dysfunction that occurs with hypercaloric nutrition. However, leptin remains a pleiotropic hormone that exerts diverse effects in a coordinated response to maintain cardiometabolic homeostasis. Greater understanding of leptin's signaling partners has been needed to fine-tune its therapeutic utilization, and our investigations were designed with that goal in mind. Preterm and growth-restricted term infants have significant reductions in circulating leptin levels, and both populations may benefit from early supplementation targeted to leptin level normalization $(34,35)$. Our results may translate most directly to the premature population who must undergo critical phases of neurodevelopment without the benefit of transplacental leptin. Exogenous leptin administration has already been shown to elicit neurotrophic effects in growth-restricted piglets and genetically leptin-deficient humans $(36,37)$. Future studies are necessary to determine if nLep can improve upon the guarded cardiovascular prognosis of premature infants. 


\section{METHODS}

\section{Animal Models}

All procedures conformed with the National Institutes of Health Guide for the Care and Use of Laboratory Animals and were approved by the Institutional Animal Care and Use Committee at the University of Iowa. Unless otherwise indicated, all chemicals and reagents were obtained from Sigma (St. Louis, MO). Pregnant C57BL/6 mice were provided a standard diet and allowed to deliver naturally. The initial cohort of mice was kept with their birth litter, and World Health Organization guidelines were utilized to define growth restriction at the tenth percentile for sex and age (38). As previously described, naturally-occurring nGR was therefore defined by a male weight $<7.1 \mathrm{~g}$ on postnatal day 20 (15). The impact of nLep on nGR was subsequently investigated by cross-fostering normal birth weight pups into litters of 6 or 12 . Those in litters of 12 were randomized to daily injections of vehicle (normal saline, $10 \mathrm{ml} / \mathrm{kg}$ ) or physiologic leptin supplementation $(80 \mathrm{ng} / \mathrm{g} / \mathrm{d}$, Biomyx Technology, San Diego, CA) on days 4-14. Our previous experiments have shown that this nLep regimen completely normalizes the circulating leptin levels of mice with incipient nGR (14). Beginning at 4 mo, adult male nGRvehicle and nGR-nLep mice from litters of 12 with weanling weights $<7.1 \mathrm{~g}$ were compared to control male mice from litters of 6 that received vehicle injections and had weanling weights $>7.1 \mathrm{~g}$.

\section{Radiotelemetry}

Carotid radiotelemetry catheters (PA-C10; Data Sciences International, St. Paul, MN) were implanted during isoflurane-induced general anesthesia (Phoenix Scientific, St. Louis, MO), as previously described (30). Flunixin meglumine $(2.5 \mathrm{mg} / \mathrm{kg}$, Phoenix Scientific) was administered subcutaneously at the time of anesthetic induction and $0.5 \%$ bupivacaine (Pfizer, New York, NY) was applied to the wound margin. After a 7 -d recovery period, arterial pressures, heart rate, and relative locomotor activity were recorded for $10 \mathrm{~s}$ every $5 \mathrm{~min}$ for $60 \mathrm{~h}$ (encompassing three dark cycles and two light cycles). Following those baseline recordings, the first cohort of natural nGR mice were utilized to investigate basal autonomic tone. On sequential days, mice received randomized injections of normal saline $(0.9 \% \mathrm{NaCl}, 10 \mathrm{ml} / \mathrm{kg})$, the muscarinic antagonist scopolamine $(2 \mathrm{mg} / \mathrm{kg})$, the nicotinic receptor antagonist chlorisondamine $(2.5 \mathrm{mg} / \mathrm{kg})$ or the $\alpha-1$ adrenergic receptor antagonist prazosin $(1 \mathrm{mg} / \mathrm{kg})$. Blood pressure and heart rate were continuously recorded throughout the 20-50 min postinjection interval of relative cardiovascular stability that follows dissipation of injection-related tachycardia and precedes loss of pharmacologic efficacy.

\section{Intracerebroventricular Cannulation}

After baseline radiotelemetry, the left cerebral ventricle was cannulated under isoflurane anesthesia (Phoenix Scientific). Flunixin meglumine $(2.5 \mathrm{mg} / \mathrm{kg}$, Phoenix Scientific) was administered subcutaneously once or twice daily for $48 \mathrm{~h}$ with the first dose given at the time of isoflurane induction, and $0.5 \%$ bupivacaine (Pfizer) was applied at the insertion site. Mice were provided $48 \mathrm{~h}$ of recovery. For the initial cohort of nGR and control mice, arterial waveforms were recorded 30-60 min after placement in a $10 \mathrm{~cm}$ diameter movementconstraining, wire-bottom metabolic cage (Hatteras Instruments, Cary, NC) utilized to elicit a reproducible stress response (39). The following day, the cage switch was repeated immediately following administration of the AT $\mathrm{R}_{1}$ antagonist losartan $(20 \mu \mathrm{g} \mathrm{icv})$.

For the second cohort of control-vehicle, nGR-vehicle and nGR-nLep mice, the icv cannula was instead connected to a subcutaneous osmotic pump (Model 1002, Alzet, Cupertino, CA) containing either aCSF or losartan $(10 \mu \mathrm{g} / \mu \mathrm{l})$ to provide a $14-\mathrm{d}$ continuous infusion at a rate of 0.25 $\mu \mathrm{l} / \mathrm{h}$ or $2.5 \mu \mathrm{g} / \mathrm{h}$, approximating the $2 \mu \mathrm{g} / \mathrm{h}$ infusion rate shown to inhibit angiotensin II-induced hypertension without altering baseline arterial pressure (40). After allowing $48 \mathrm{~h}$ for surgical recovery and stabilization, hemodynamic parameters were collected on days 3 through 6 of icv infusion (icv baseline). On day 7 of icv infusion, mice were placed in the aforementioned metabolic cage to assess the effect of chronic losartan administration on stress reactivity (39). On day 8 of icv infusion, vehicle was administered $(0.9 \% \mathrm{NaCl}, 10 \mathrm{ml} / \mathrm{kg}$ intraperitoneal), and this was followed on day 9 by administration of a single dose of leptin $(1 \mathrm{mg} /$ $\mathrm{kg}$ intraperitoneal, Biomyx Technology). On days 10 through 13, while the mice continued to receive aCSF vs. losartan, hemodynamics were recorded during twice daily intraperitoneal leptin administration (1 mg/ kg, Biomyx Technology).

\section{Body Weight and Food Intake}

During a continuous $72 \mathrm{~h}$ window that fully encompassed the baseline radiotelemetry window proceeding icv cannulation, food intake was calculated as the difference between initial food weight and final food weight. Mouse weight was obtained at the end of the recording window, and the weight of the radiotransmitter $(1.4 \mathrm{~g})$ was subtracted. The process was repeated during the second baseline radiotelemetry epoch during which time the mice were receiving a continuous infusion of icv aCSF or losartan (baseline two). The third and final recording interval occurred during twice daily intraperitoneal leptin administration.

\section{Corticosterone ELISA}

To verify the presence of a physiologic stress response additional adult control and nGR mice had serial blood sampling at baseline and $1 \mathrm{~h}$ after placement in the metabolic cage. Plasma was stored at $-80^{\circ} \mathrm{C}$ until corticosterone analysis was completed by enzyme-linked immunosorbent assay (ELISA) (Immunodiagnostic Systems, Tyne and Wear, UK).

\section{RSNA}

Mice were initially anesthetized with intraperitoneal ketamine $(91 \mathrm{mg} /$ kg, JHP Pharmaceuticals, Rochester, MN) plus xylazine $(9.1 \mathrm{mg} /$ $\mathrm{kg}$, Akorn Pharmaceuticals, Decatur, IL) or isoflurane (Phoenix Scientific) and then sustained throughout the experiment with infusion of $\alpha$-chloralose $(25 \mathrm{mg} / \mathrm{kg}$ followed by $6 \mathrm{mg} / \mathrm{kg} / \mathrm{h}$ ) via a right jugular vein catheter. A polyethylene (PE-50) tubing was used to intubate the mouse for spontaneous respiration of oxygen-enriched room air. Finally, continuous blood pressure was measured from an indwelling left carotid artery catheter. The left renal nerve was exposed and a 36-gauge platinum-iridium recording electrode was sealed to the renal nerve bundle. After $1-3 \mathrm{~h}$, a stable baseline for blood pressure and renal nerve activity were obtained and the mice received intravenous leptin ( $1 \mathrm{mg} / \mathrm{kg}$, Biomyx Technology). After $2 \mathrm{~h}$ of RSNA/ hemodynamic recording, the mice received a single intravenous injection of the nitric oxide synthase antagonist L-NAME $(25 \mathrm{mg} / \mathrm{kg})$ and hemodynamic recordings were continued an additional $10 \mathrm{~min}$, at which point the mice were euthanized.

\section{Real-Time Quantitative Reverse Transcription Polymerase Chain Reaction}

After overnight fast, the brain was rapidly harvested and frozen in isopentane held at the temperature of crushed dry ice followed by immediate storage at $-80^{\circ} \mathrm{C}$. Individual regions were harvested by first cryosectioning the hypothalamus into $300 \mu \mathrm{m}$ sections onto glass slides. The sections were kept frozen while the ARC and ventromedial hypothalamus were isolated using core biopsy needles under stereomicroscopic examination. RNA was purified from these samples using TRIzol (Thermo Fisher Scientific, Waltham, MA) and RNeasy kit (Qiagen, Valencia, CA). All gene expression experiments employed TaqMan primer/probe real-time polymerase chain reaction assays (Thermo Fisher Scientific). The catalog numbers for the specific assays were as follows: GAPDH 4352932E; long form of the leptin receptor (LepRb) Mm01265583_m1; short form of the leptin receptor (LepRa) Mm01262070_m1; AT R Mm01166161_m1. Relative gene expression was determined by the $2^{-\Delta \Delta C T}$ method after excluding values with $\Delta C_{t}$ values 1.5 times the interquartile range greater than the third quartile or 1.5 times the interquartile range less than the first quartile.

\section{Data Analysis}

Values are presented as mean \pm SE. Because weanling weights were used to categorize all mice as either nGR or control, one-way ANOVA was used to analyze between group differences with Holm-Šídák correction for multiple comparisons. For the remainder of the data, in order to test the a priori hypothesis that only nGR mice that did not receive nLep would differ from control mice, those two groups were first compared by ANOVA with the Holm-Š́lák method used to correct for multiple comparisons. One-way ANOVA was used for all baseline data and two-way ANOVA was used for nGR vs. control mice receiving aCSF vs. losartan. If two-way ANOVA identified a significant interaction between the factors, one-way ANOVA was used to 
independently evaluate for between group effects. Two-way repeated measures ANOVA was used to analyze the response to stress, intraperitoneal injection or icv injection with one factor being nGR vs. control and the other factor baseline vs. stress, baseline vs. injection or stress vs. injection. Two-way repeated measures ANOVA was likewise used to analyze leptin-induced renal sympathetic and cardiovascular responses with factoring for nGR and elapsed time. A value of $P<0.05$ was considered significant. Whenever significant differences between nGR-saline and control mice were identified, we tested the hypothesis that nGR-nLep mice did not differ from control mice at those specific endpoints. For this equivalency testing, the equivalence margin $(\delta)$ was set to equal the observed absolute difference in the means of the nGR-saline and control mice. Using the standard two one-sided test procedure, equivalence between the nGR-nLep and control groups was established at the $\alpha=0.05$ significance level if the $90 \%$ confidence interval for the difference in their means was contained within the interval $(-\delta, \delta)$. All analyses were performed using SigmaStat 3.0x (SPSS, Chicago, IL).

\section{STATEMENT OF FINANCIAL SUPPORT}

This study was supported by National Institutes of Health, Bethesda, MD (HL007485, HL084207, HL102659).

\section{Disclosure: There is no conflict of interest to disclose.}

\section{REFERENCES}

1. World Health Organization. Low Birth Weight: A Tabulation of Available Information, WHO/MCH/92.2. World Health Organization, Geneva and UNICEF, New York, 1992.

2. Dagle JM, Fisher TJ, Haynes SE, et al. Cytochrome P450 (CYP2D6) genotype is associated with elevated systolic blood pressure in preterm infants after discharge from the neonatal intensive care unit. J Pediatr 2011;159:104-9.

3. Dusick AM, Poindexter BB, Ehrenkranz RA, Lemons JA. Growth failure in the preterm infant: can we catch up? Semin Perinatol 2003;27:302-10.

4. Johansson S, Iliadou A, Bergvall N, Tuvemo T, Norman M, Cnattingius S. Risk of high blood pressure among young men increases with the degree of immaturity at birth. Circulation 2005;112:3430-6.

5. Simonds SE, Pryor JT, Ravussin E, et al. Leptin mediates the increase in blood pressure associated with obesity. Cell 2014;159:1404-16.

6. Briffa JF, McAinch AJ, Romano T, Wlodek ME, Hryciw DH. Leptin in pregnancy and development: a contributor to adulthood disease? Am J Physiol Endocrinol Metab 2015;308:E335-50.

7. Kawamata R, Suzuki Y, Yada Y, et al. Gut hormone profiles in preterm and term infants during the first 2 months of life. J Pediatr Endocrinol Metab 2014;27:717-23.

8. Spear ML, Hassink SG, Leef K, et al. Immaturity or starvation? Longitudinal study of leptin levels in premature infants. Biol Neonate 2001;80:35-40.

9. Sipola-Leppänen $M$, Väräsmäki $M$, Tikanmäki $M$, et al. Cardiometabolic risk factors in young adults who were born preterm. Am J Epidemiol 2015;181:861-73.

10. Allison MA, Ix JH, Morgan C, et al. Higher leptin is associated with hypertension: the Multi-Ethnic Study of Atherosclerosis. J Hum Hypertens 2013;27:617-22.

11. Dubinion JH, do Carmo JM, Adi A, Hamza S, da Silva AA, Hall JE. Role of proopiomelanocortin neuron Stat3 in regulating arterial pressure and mediating the chronic effects of leptin. Hypertension 2013;61:1066-74.

12. Hilzendeger AM, Morgan DA, Brooks L, et al. A brain leptin-renin angiotensin system interaction in the regulation of sympathetic nerve activity. Am J Physiol Heart Circ Physiol 2012;303:H197-206.

13. Grove KL, Grayson BE, Glavas MM, Xiao XQ, Smith MS. Development of metabolic systems. Physiol Behav 2005;86:646-60.

14. Erkonen GE, Hermann GM, Miller RL, et al. Neonatal leptin administration alters regional brain volumes and blocks neonatal growth restrictioninduced behavioral and cardiovascular dysfunction in male mice. Pediatr Res 2011;69(5 Pt 1):406-12.

15. Hermann GM, Miller RL, Erkonen GE, et al. Neonatal catch up growth increases diabetes susceptibility but improves behavioral and cardiovascular outcomes of low birth weight male mice. Pediatr Res 2009;66:53-8.

16. Meyer LR, Zhu V, Miller A, Roghair RD. Growth restriction, leptin, and the programming of adult behavior in mice. Behav Brain Res 2014;275:131-5.
17. Johansson S, Norman M, Legnevall L, Dalmaz Y, Lagercrantz H, Vanpée $M$. Increased catecholamines and heart rate in children with low birth weight: perinatal contributions to sympathoadrenal overactivity. J Intern Med 2007;261:480-7.

18. Morgan DA, Thedens DR, Weiss R, Rahmouni K. Mechanisms mediating renal sympathetic activation to leptin in obesity. Am J Physiol Regul Integr Comp Physiol 2008;295:R1730-6.

19. Eriksson JG, Forsén TJ, Kajantie E, Osmond C, Barker DJ. Childhood growth and hypertension in later life. Hypertension 2007;49:1415-21.

20. Pladys P, Sennlaub F, Brault S, et al. Microvascular rarefaction and decreased angiogenesis in rats with fetal programming of hypertension associated with exposure to a low-protein diet in utero. Am J Physiol Regul Integr Comp Physiol 2005;289:R1580-8.

21. Dodic M, McAlinden AT, Jefferies AJ, et al. Differential effects of prenatal exposure to dexamethasone or cortisol on circulatory control mechanisms mediated by angiotensin II in the central nervous system of adult sheep. J Physiol 2006;571(Pt 3):651-60.

22. Roghair RD, Aldape G. Naturally occurring perinatal growth restriction in mice programs cardiovascular and endocrine function in a sex- and straindependent manner. Pediatr Res 2007;62:399-404.

23. Pyhälä R, Räikkönen K, Feldt K, et al. Blood pressure responses to psychosocial stress in young adults with very low birth weight: Helsinki study of very low birth weight adults. Pediatrics 2009;123:731-4.

24. Lee DL, Webb RC, Brands MW. Sympathetic and angiotensin-dependent hypertension during cage-switch stress in mice. Am J Physiol Regul Integr Comp Physiol 2004;287:R1394-8.

25. Crume TL, Scherzinger A, Stamm E, et al. The long-term impact of intrauterine growth restriction in a diverse U.S. cohort of children: the EPOCH study. Obesity (Silver Spring) 2014;22:608-15.

26. Hack M, Schluchter M, Cartar L, Rahman M, Cuttler L, Borawski E. Growth of very low birth weight infants to age 20 years. Pediatrics 2003;112(1 Pt 1): $\mathrm{e} 30-8$.

27. Porter JP, Potratz KR. Effect of intracerebroventricular angiotensin II on body weight and food intake in adult rats. Am J Physiol Regul Integr Comp Physiol 2004;287:R422-8.

28. Jones A, Beda A, Ward AM, et al. Size at birth and autonomic function during psychological stress. Hypertension 2007;49:548-55.

29. Kuo JJ, Jones OB, Hall JE. Inhibition of NO synthesis enhances chronic cardiovascular and renal actions of leptin. Hypertension 2001;37(2 Pt 2):670-6.

30. Roghair RD, Segar JL, Volk KA, et al. Vascular nitric oxide and superoxide anion contribute to sex-specific programmed cardiovascular physiology in mice. Am J Physiol Regul Integr Comp Physiol 2009;296:R651-62.

31. Harlan SM, Morgan DA, Agassandian K, et al. Ablation of the leptin receptor in the hypothalamic arcuate nucleus abrogates leptin-induced sympathetic activation. Circ Res 2011;108:808-12.

32. Münzberg H, Flier JS, Bjørbaek C. Region-specific leptin resistance within the hypothalamus of diet-induced obese mice. Endocrinology 2004;145:4880-9.

33. Luckett BS, Frielle JL, Wolfgang L, Stocker SD. Arcuate nucleus injection of an anti-insulin affibody prevents the sympathetic response to insulin. Am J Physiol Heart Circ Physiol 2013;304:H1538-46.

34. Pighetti M, Tommaselli GA, D'Elia A, et al. Maternal serum and umbilical cord blood leptin concentrations with fetal growth restriction. Obstet Gynecol 2003;102:535-43.

35. Su PH, Wang SL, Chen JY, Lai CP, Jian SH. Serum leptin levels in preterm, healthy and sick-term newborns. Acta Paediatr Taiwan 2002;43:249-54.

36. Attig L, Djiane J, Gertler A, et al. Study of hypothalamic leptin receptor expression in low-birth-weight piglets and effects of leptin supplementation on neonatal growth and development. Am J Physiol Endocrinol Metab 2008;295:E1117-25.

37. Matochik JA, London ED, Yildiz BO, et al. Effect of leptin replacement on brain structure in genetically leptin-deficient adults. J Clin Endocrinol Metab 2005;90:2851-4.

38. WHO 1995 Physical status: the use and interpretation of anthropometry. Report of a WHO Expert Committee. World Health Organ Tech Rep Ser 854:1-452.

39. Roghair RD, Wemmie JA, Volk KA, Scholz TD, Lamb FS, Segar JL. Maternal antioxidant blocks programmed cardiovascular and behavioural stress responses in adult mice. Clin Sci (Lond) 2011;121:427-36.

40. Grobe JL, Grobe CL, Beltz TG, et al. The brain Renin-angiotensin system controls divergent efferent mechanisms to regulate fluid and energy balance. Cell Metab 2010;12:431-42. 